\title{
OBTAINING APPROXIMATE VALUES OF EXTERIOR ORIENTATION ELEMENTS OF MULTI-INTERSECTION IMAGES USING PARTICLE SWARM OPTIMIZATION
}

\author{
X. Li ${ }^{\mathrm{a}}, \mathrm{S} . \mathrm{W} . \mathrm{Li}^{\mathrm{a}, *}$ \\ ${ }^{a}$ School of Remote Sensing and Information Engineering, Wuhan University, 129 Luoyu Road Wuhan, China - \\ xli2126@whu.edu.cn, lishuwen0605@163.com
}

Commission III, WG III/1

KEY WORDS: Pose estimation, Approximate value, Exterior orientation elements, Particle swarm optimization, Small control frame

\begin{abstract}
:
In this paper, an efficient global optimization algorithm in the field of artificial intelligence, named Particle Swarm Optimization (PSO), is introduced into close range photogrammetric data processing. PSO can be applied to obtain the approximate values of exterior orientation elements under the condition that multi-intersection photography and a small portable plane control frame are used.
\end{abstract}

PSO, put forward by an American social psychologist J. Kennedy and an electrical engineer R.C. Eberhart, is a stochastic global optimization method based on swarm intelligence, which was inspired by social behavior of bird flocking or fish schooling.

The strategy of obtaining the approximate values of exterior orientation elements using PSO is as follows: in terms of image coordinate observed values and space coordinates of few control points, the equations of calculating the image coordinate residual errors can be given. The sum of absolute value of each image coordinate is minimized to be the objective function. The difference between image coordinate observed value and the image coordinate computed through collinear condition equation is defined as the image coordinate residual error. Firstly a gross area of exterior orientation elements is given, and then the adjustment of other parameters is made to get the particles fly in the gross area. After iterative computation for certain times, the satisfied approximate values of exterior orientation elements are obtained. By doing so, the procedures like positioning and measuring space control points in close range photogrammetry can be avoided. Obviously, this method can improve the surveying efficiency greatly and at the same time can decrease the surveying cost. And during such a process, only one small portable control frame with a couple of control points is employed, and there are no strict requirements for the space distribution of control points.

In order to verify the effectiveness of this algorithm, two experiments are carried out. In the first experiment, images of a standard grid board are taken according to multi-intersection photography using digital camera. Three points or six points which are located on the left-down corner of the standard grid are regarded as control points respectively, and the exterior orientation elements of each image are computed through PSO, and compared with these elements computed through bundle adjustment. In the second experiment, the exterior orientation elements obtained from the first experiment are used as approximate values in bundle adjustment and then the space coordinates of other grid points on the board can be computed. The coordinate difference of grid points between these computed space coordinates and their known coordinates can be used to compute the accuracy. The point accuracy computed in above experiments are $\pm 0.76 \mathrm{~mm}$ and $\pm 0.43 \mathrm{~mm}$ respectively. The above experiments prove the effectiveness of PSO used in close range photogrammetry to compute approximate values of exterior orientation elements, and the algorithm can meet the requirement of higher accuracy.

In short, PSO can get better results in a faster, cheaper way compared with other surveying methods in close range photogrammetry.

\footnotetext{
* Corresponding author.
} 


\section{INTRODUCTION}

As a non-contact measurement method, close-range photogrammetry has been used in many fields, such as industrial field, biomedical field, etc. Admittedly, the generally accepted way in close-range photogrammetry is to firstly set up some control points around the object or on the surface of object when the images of object are taken according to multiintersection photography mode. Then the exterior orientation elements can be calculated using the space coordinates and image coordinates of these control points. And in the rest bundle adjustment, the exterior orientation elements are employed as the initial values and recalculated, and space coordinates of unknown points are also calculated at the same time.

There are several typical methods, namely Space Resection, Pyramid Principle and Direct Linear Transformation etc., to compute exterior orientation elements in close-range photogrammetry (Feng, 2003). Wang put forward a method to slove approximate values of exterior orientation elements based on 4 points on a same plane(Wang, 2006), and 2D direct linear transformation is used to calculate approximate values of exterior orientation elements (Zhang, 2002), and Guan Yunlan etc. raise a space resection method based on unit quaternion (Guan, 2008). These methods will be invalid if a small portable plane control frame with few control points. Haralick summarizes all prior work on the minimal solution for the absolute orientation problem and recommends a solution which is numerically stable (Haralick, 1994).

Through researching bird flocking, Reynolds found that a bird only trace its limited number neighbour birds and want to find food. But the final result is that the whole bird flock is likely controlled under a same center. Namely, when a bird flock is looking for food, the simplest way for the bird flock is to seek the birds who are nearest to the food. Particle Swarm Optimization (PSO) was put forward by an American social psychologist Kennedy, J. and an electrical engineer Eberhart, R.C., is a stochastic global optimization method based on swarm intelligence, which was inspired by social behavior of bird flocking or fish schooling (Kennedy, 1995).

Based on the strong global search feature of PSO, a improved PSO method is put forward in this paper to calculate approximate values of exterior orientation elements of closerange images in this paper.

\section{PARTICLE SWARM OPTIMIZATION ALGORITHM}

For example, suppose there is a food (the black triangle) in a plane, shown as in Figure 1(a). It is located at the center in the area, which are $X, Y \in[0,200]$. At the beginning, twenty birds(shown as the black points) randomly fly without object in the area. When the bird flock find the food in the area, almost all birds will fly to the food point through certain time flying, shown as in Figure 1(b)(c).

PSO for solving a optimization problem, a bird is called as particle or agent, a solution of the problem corresponds to a bird's position in search space. The fitness of all of the particles is determined by a objective function, and the flying direction and distance of each particle are defined by its' flying speed. Then the particles will trace the current optimal particle and search for the optimization in the solution space

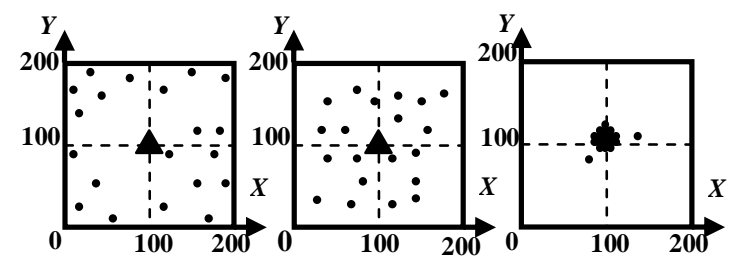

(a)

(b)

(c)

Figure 1. Stimulation of birds feeding

(a) initial distribution of birds (b) distribution of birds after 20 iteration (c) distribution of birds after 100 iteration

At the beginning of PSO procedure, a swarm of particle (random solution) are initialized, and the optimal solutions will be found through evolutionary computation (iterative computation). Each particle will update themselves through tracing two extremum. One extremum, called individual extremum $p_{B e s t}$, is the optimal position found by the particle itself, the other, called globe extremum $g_{\text {Best }}$, is the optimal position found by the whole particle swarm.

The mathematical description of PSO is flowed. Suppose in a D-dimensional search space, the total number of particles is $n$. Vector $x_{i}=\left(x_{i 1}, x_{i 2} \ldots x_{i D}\right)$ means the position of particle $i$. $p_{\text {Best }}=\left(p_{i 1}, p_{i 2} \ldots p_{i D}\right)$ means the optimal position which is searched by particle $i$ till present step. $g_{\text {Best }}=\left(g_{1}, g_{2} \ldots g_{D}\right)$ means the optimal position which is searched by the whole particle swarm till present step. The position changing rate(speed) of particle $i$ is shown as $v_{i}=\left(v_{i 1}, v_{i 2} \ldots v_{i D}\right)$. So the speed and position of particle $i$ are described as equation (1).

$$
\left\{\begin{array}{l}
v_{i d}(t+1)=G_{1}+G_{2}+G_{3} \\
x_{i d}(t+1)=x_{i d}(t)+v_{i d}(t+1) \quad 1 \leq i \leq n
\end{array}\right.
$$

Where, $G_{1}=v_{i d}(t)$, is the former speed item, and expresses the influence of former speed to present speed of particle $i$;

$G_{2}=c_{1} r_{1}\left(p_{i d}(t)-x_{i d}(t)\right)$, is the self-cognition item, and means the influence of the historical optimal position to present position of particle $i$ while only the experience of particle $i$ is considered; $G_{3}=c_{2} r_{2}\left(p_{g d}(t)-x_{i d}(t)\right)$, is the social sharing of information item, and indicates the influence of the historical optimal position of the whole particle swarm to present position of particle $i ; c_{1}, c_{2}$ are acceleration factors. $c_{1}$ is used to adjust the particle flying step size toward itself optimal position. $c_{2}$ is used to adjust the particle flying step length toward global optimal position. $r_{1}, r_{2}$ are two random numbers belong to $[0,1] . x_{i d}$ is the position in No. $d$ dimensional for particle $i$ at No.t iterative. $p_{i d}$ is the individual extremum position in No.d dimensional for particle $i$. $p_{g d}$ is the globe extremum position in No.d dimensional for the whole particle swarm.

The initial position and initial speed of particle swarm are generated randomly, and then the procedure goes into iterative computation according to equation(1) till the satisfactory solution is found. In equation(1) item $G_{1}$ can ensure the globe convergence performance, and $G_{2}$ and $G_{3}$ are employed to ensure the local convergence performance (Zeng, 2004). 


\section{IMPROVED PARTICLE SWARM OPTIMIZATION WITH INERTIA WEIGHT FACTOR}

There is the advantage of fast convergence for PSO, but the disadvantage also is lower accuracy. For PSO algorithm, if acceleration factors and maximum speed are too high, particles may fly over optimal solution and the result is not converge. However, under the convergent situation, because all of particles fly to the direction of optimal solution, those particles will lose diversity, and the convergent speed of algorithm will be obviously slow down later. In order to solve the problem, Shi etc. put forward improved particle swarm optimization with inertia weight factor (Shi, 1998), whose equation is:

$$
\left\{\begin{array}{l}
v_{i d}(t+1)=w \cdot G 1+G 2+G 3 \\
x_{i d}(t+1)=x_{i d}(t)+v_{i d}(t+1) \quad 1 \leq i \leq n
\end{array}\right.
$$

Where $w$ is inertia weight factor which relates to former speed. Its function is to control the influence of former speed on present speed. Larger $w$ value can strengthen global search capability of PSO, while smaller $w$ value can strengthen local search capability of PSO (Parsopoulos, 2001). This improved method can speed up converging and increase the effect of PSO algorithm.

Shi suggests $w$ be within [0 1.4]. However, the experiment result shows that when $w$ is within [0.8 1.2], converging could be much faster, whereas when $w>1.2$, the algorithm will be more likely stuck in local extremum (Shi, 1998). The usual way to set $w$ is below (Shi, 1999).

$$
w=w_{\max }-\left(w_{\max }-w_{\min }\right) \cdot r u n / r u n_{\max }
$$

Where, $w_{\max }, w_{\min }$ are respectively maximum inertia weight, minimum inertia weight, run is present number of iteration, run $_{\max }$ is maximum number of iteration, which could be interpreted as inertia weight factor $w$ could be taken as the function of run. By the testing result (Shi, 1999), the insertion of inertia weight factor can bring about better effect.

\section{STRATEGY AND PROCEDURE OF CALCULATING APPROXIMATE VALUES OF EXTERIOR ORIENTATION ELEMENTS BASED ON PSO}

In order to increase the speed and accuracy of calculating the approximate values of exterior orientation elements using PSO, the method of limiting the number of searching is applied. Through many experiments, the maximum searching number is set as 500 .

In object space coordinate system $O-X Y Z$, point $S$ is projection center, and $C P_{i}(i=1,2 \ldots n)$ is object control point, shown in Figure 2.

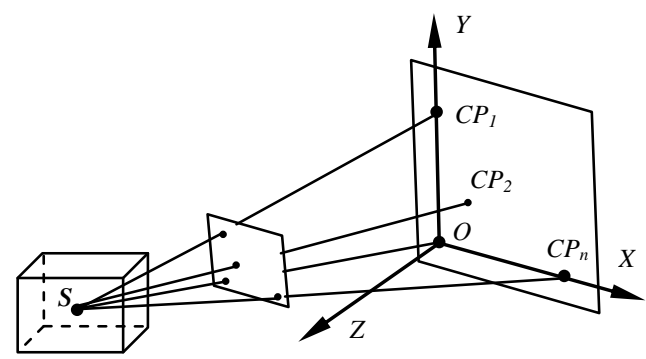

Figure 2. Diagram of Photography

The flow chart of calculating the approximate values of exterior orientation elements of a image using PSO is shown as Figure 3.

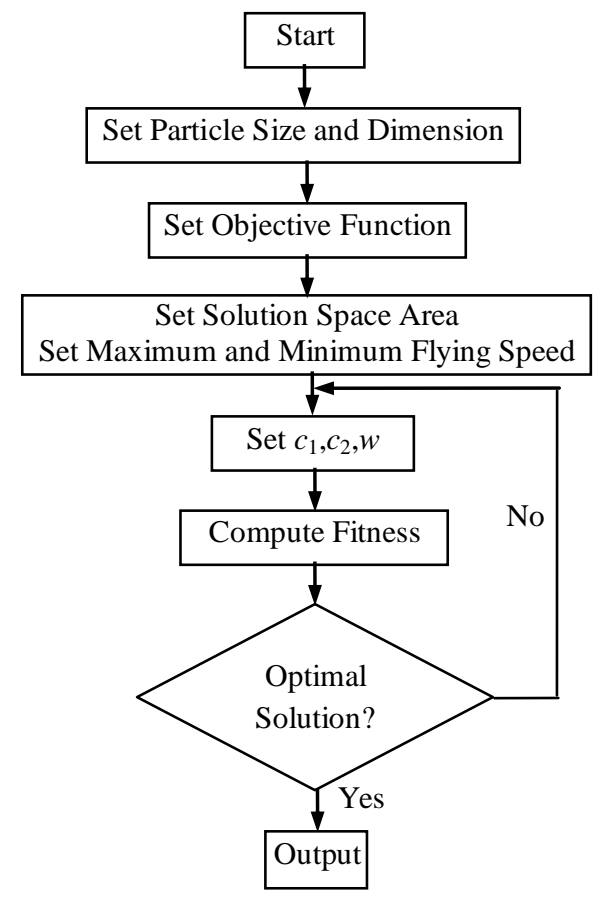

Figure 3. Flow chart of calculating the approximate values of exterior orientation elements of a single image using PSO

\subsection{Define of Objective Function}

$n(n \geq 3)$ object control points are employed in PSO. Image point coordinate Residual errors $\left(v_{x i}, v_{y} i\right)$ of each control point can be calculated. Minimum Sum of residual errors absolute value of all image points of control points in this image is regarded as the objective function, shown as in equation(4).

$$
\min \quad V=\sum_{i=1}^{n}\left|v_{i}\right| \quad(i=1,2 \ldots n, n \geq 3)
$$

where, $\left|v_{i}\right|=\left|v_{x i}\right|+\left|v_{y i}\right| ;\left|v_{x i}\right|$ is the residual error absolute value of $x$ coordinate of image point $i$ which corresponds to object control point $C P_{i}$, and $\left|v_{y i}\right|$ is the residual error absolute value of $y$ coordinate of image point $i$ which corresponds to object control point $C P_{i}$, and $v_{x i}, v_{y i}$ can be expressed as: 


$$
\left\{\begin{array}{l}
v_{x i}=x_{i}-x_{0}+\Delta x_{i}-f \frac{a_{1}\left(X_{i}-X_{S}\right)+b_{1}\left(Y_{i}-Y_{S}\right)+c_{1}\left(Z_{i}-Z_{S}\right)}{a_{3}\left(X_{i}-X_{S}\right)+b_{3}\left(Y_{i}-Y_{S}\right)+c_{3}\left(Z_{i}-Z_{S}\right)} \\
v_{y i}=y_{i}-y_{0}+\Delta y_{i}-f \frac{a_{2}\left(X_{i}-X_{S}\right)+b_{2}\left(Y_{i}-Y_{S}\right)+c_{2}\left(Z_{i}-Z_{S}\right)}{a_{3}\left(X_{i}-X_{S}\right)+b_{3}\left(Y_{i}-Y_{S}\right)+c_{3}\left(Z_{i}-Z_{S}\right)}
\end{array}\right.
$$

Where $\left(x_{i}, y_{i}\right)$ is image coordinates of point $i$, and $\Delta x_{i}, \Delta y_{i}$ are distortion correction of image point $i$.

\subsection{Setting of Number and Dimension of Particle}

When Particle Size is bigger than 60, the successful converging is more likely to happen; the increase in the population scale scan increase the stability of algorithm, whereas the calculating time will be more (Wei, 2008). Based on the amount of experiments, the author suggests setting range of the particle size between 40 and 50 .

The position of each particle represents a solution. Therefore, the dimension of solution vector decides the dimension of particle. Because there are six parameters, which could be taken as 6-dimensional vector, the dimension of particle in one image mode must be 6 .

\subsection{Setting solution space range and maximum and minimum flying speed}

4.3.1 Setting of Solution Space Range: In the procedure of solving the approximate values of exterior orientation elements, the searching area of exterior orientation elements should be set, as the cube shown in Figure2.

In the gross estimation of the position of exterior orientation elements, the solution space range is decided by the number of control points, or rather the gross range of line elements, angle elements. The more the control points, the wider the set range of solution space, and conversely, the fewer, the narrower. The experiments shows: if 3 object control points are used, the range of line elements of exterior orientation elements is set by line element standard value $\pm 1 \mathrm{~m}$, and the angle element range of exterior orientation elements is set by angle element standard value \pm 0.5 radian; if 6 object control points are used, the range of line elements of exterior orientation elements is set by line element standard value $\pm 5 \mathrm{~m}$, and the angle element range of exterior orientation elements is set by angle element standard value \pm 1 radian. Here, the exterior orientation elements calculated by bundle adjustment are regarded as the standard value. Under this condition, the approximate values of exterior orientation elements calculated by PSO can be used in bundle adjustment.

4.3.2 Setting of the Maximum and Minimum Flying Speed: The flying speed of particles decides the speed and quality of searching. If the speed is too high, the optimal solution couldn't be available; if the speed is too low, the searching area of particles would be too narrow and the possibility of finding optimal solution is also very low. That would lead to local optimization. The maximum and minimum flying speed is expressed as below:

$$
\left\{\begin{array}{l}
v_{d \text { max }}=k\left(x_{d \text { max }}-x_{d \text { min }}\right) \\
v_{d \text { min }}=-k\left(x_{d \text { max }}-x_{d \text { min }}\right)
\end{array}\right.
$$

where $v_{d \max }, v_{d \min }$ are the particle maximum and minimum flying speed in d-dimensional vector, and $x_{d \max }, x_{d \min }$ are the maximum and minimum limited range for particle in $\mathrm{d}$ dimension vector, and $k$ is a scale factor. In general, the value of $\mathrm{k}$ is between $0.1 \sim 0.5$. In this paper, the value of $\mathrm{k}$ which fits for solving of the approximate values of exterior orientation elements using PSO equal to 0.1 approximately.

\subsection{Setting of Acceleration Factors $c_{1}, c_{2}$}

In this paper, $c_{1}, c_{2}$ are set as 2 , which $c_{1}$ is a important parameter in self-cognition item $G_{2}$, and $c_{2}$ also is a important parameter in the social sharing of information item $G_{3}$.

\subsection{Setting of Inertia Weight Factor}

In this paper, the algorithm is improved based on the strategy of linear decreasing weight, and inertia weight factor $w$ is determined by equation(6) and (7).

$$
\begin{gathered}
w_{0}=1-r u n / \text { run }_{\text {Max }} \\
w= \begin{cases}0.6 & \left(w_{0} \geq 0.6\right) \\
w_{0} & \left(0.4<w_{0}<0.6\right) \\
0.4 & w_{0} \leq 0.4\end{cases}
\end{gathered}
$$

Some constants used in PSO is set as in Table 1.

\begin{tabular}{|c|c|c|c|}
\hline Parameter & Value & Parameter & Value \\
\hline Particle size & 45 & $c_{1}$ & 2.0 \\
\hline Particle dimension & 6 & $c_{2}$ & 2.0 \\
\hline$k$ & \multicolumn{2}{|c|}{0.12} \\
\hline $\begin{array}{c}\text { Residual } \\
\text { threshold(pixel) }\end{array}$ & $\begin{array}{l}(2-5) \times \text { number of control } \\
\text { points }\end{array}$ \\
\hline$w$ & $\begin{cases}0.6 & \left(w_{0} \geq 0.6\right) \\
w_{0} & \left(0.4<w_{0}<0.6\right) \\
0.4 & \left(w_{0} \leq 0.4\right)\end{cases}$ \\
& $\begin{array}{c}w_{0}=1-\frac{\text { run }}{\text { run }_{\max }} \\
\left(\text { run }_{\max }=500\right)\end{array}$ \\
\hline
\end{tabular}

Table 1. Setting of PSO Parameter

where, $w_{\text {Max }}=0.6$ and $w_{\text {Min }}=0.4$ are based on many experiments.

\subsection{Setting of Termination Condition}

Satisfactory solution will be found out through a lot of iterative calculation in PSO. In this paper, the maximum number of iteration and the threshold of sum of residual errors absolute value of all image points of control points in a image are set, and the sum of residual errors absolute value of image points is calculated using the approximate values of exterior orientation elements searched by PSO. If the difference between the sum and the threshold is less than a certain number or the number of iterations is more than the maximum number of iteration, the calculation procedure will be end. 


\section{EXPERIMENTS}

There are two experiments in this paper. In these experiments, a small 2D plane control brand is employed. Its size is $350 \mathrm{~mm} \times 350 \mathrm{~mm}$, and there are 32 code points, which the distance of every pairs of adjacent points is $70 \mathrm{~mm}$ in horizonal and vertical direction. The selected three or six points(in rectangles)located at the left-up corner are regarded as the control points of a small control frame, and the other points(in circles) are regarded as check points, shown as in Figure 4.

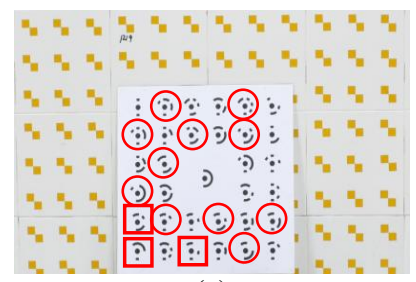

(a)

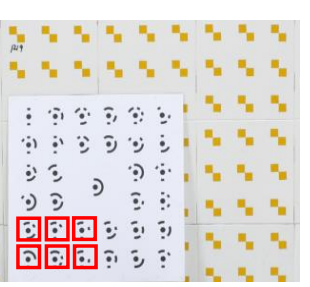

(b)
Figure 4. Diagram of control points and check points distribution (a) three control points and check points (b) six control points

A Canon 450D SLR digital camera with $28 \mathrm{~mm}$ lens is used to take six multi-intersection images which have $4272 \times 2848$ pixels. The top right number on each image expresses the image number, shown as in Figure 5. PSO algorithm is utilized to calculate the approximate values of exterior orientation elements which will be employed in bundle adjustment to compute the object space coordinates of check points. Then we compare the computed the object space coordinates of check points with their known object space coordinates to compute accuracy.

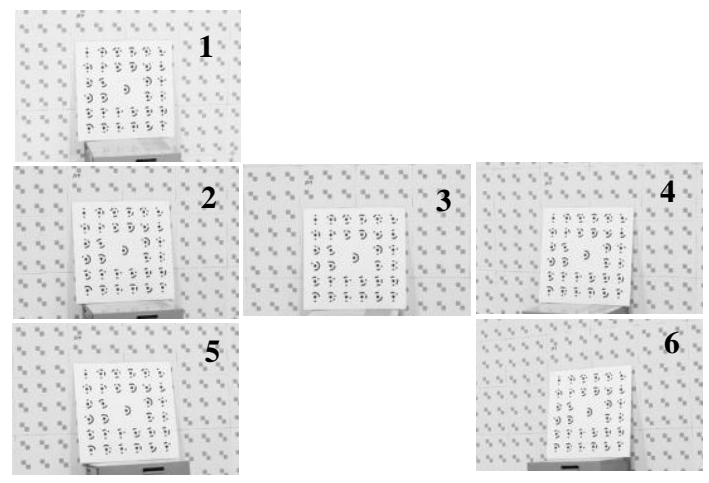

Figure 5. Six multi-intersection images

\subsection{Experiment 1. using three control points}

Six images (shown in Figure 5.) are taken and their approximate values of exterior orientation elements are searched by PSO respectively. Twelve code points are selected as check points in the control brand, shown as in Figure 4(a). The exterior orientation elements calculated by PSO and Bundle adjustment are shown in Table 2. The difference of check points object coordinates are shown in Table 3.

\begin{tabular}{|c|c|r|r|r|r|r|r|}
\hline No & Method & $X_{S}(\mathrm{~mm})$ & $Y_{S}(\mathrm{~mm})$ & $Z_{S}(\mathrm{~mm})$ & $\begin{array}{c}\varphi \\
(\text { radian })\end{array}$ & $\begin{array}{c}\omega \\
\text { (radian) }\end{array}$ & $\begin{array}{c}\kappa \\
\text { (radian) }\end{array}$ \\
\hline \multirow{2}{*}{1} & Bundle & 69.99 & 201.85 & 2473.38 & 0.04410 & -0.02370 & -0.03050 \\
\cline { 2 - 8 } & PSO & -440.71 & 254.53 & 3223.20 & 0.22203 & 0.04615 & -0.02440 \\
\hline \multirow{2}{*}{2} & Bundle & 72.07 & 145.46 & 2430.21 & 0.03140 & -0.00070 & -0.03960 \\
\cline { 2 - 8 } & PSO & -481.95 & -105.09 & 3156.94 & 0.23223 & 0.15709 & -0.05091 \\
\hline \multirow{3}{*}{3} & Bundle & 597.94 & -23.307 & 2349.79 & -0.16210 & 0.07060 & -0.00260 \\
\cline { 2 - 8 } & PSO & 408.84 & -105.23 & 3189.49 & -0.03093 & 0.15973 & -0.00643 \\
\hline \multirow{3}{*}{4} & Bundle & 1003.19 & -23.556 & 2403.45 & -0.34150 & 0.06340 & 0.04710 \\
\cline { 2 - 8 } & PSO & 1387.67 & -97.15 & 3092.11 & -0.35570 & 0.15239 & 0.05028 \\
\hline \multirow{2}{*}{5} & Bundle & -311.44 & -248.88 & 2362.60 & 0.20010 & 0.15760 & -0.06770 \\
\cline { 2 - 8 } & PSO & -545.50 & -460.54 & 3079.83 & 0.26537 & 0.26431 & -0.08714 \\
\hline \multirow{2}{*}{6} & Bundle & 1257.26 & -304.67 & 2479.63 & -0.40820 & 0.17140 & 0.02230 \\
\cline { 2 - 8 } & PSO & 1700.98 & -470.30 & 3130.53 & -0.42452 & 0.26081 & 0.03090 \\
\hline
\end{tabular}

Table 2. Exterior orientation elements calculated by PSO and Bundle adjustment (three control points)

\begin{tabular}{|c|c|c|c|c|c|c|c|}
\hline \multirow[t]{2}{*}{ No } & \multicolumn{3}{|c|}{$\begin{array}{l}\text { Difference of coordinate } \\
\qquad(\mathrm{mm})\end{array}$} & \multirow[t]{2}{*}{ No } & \multicolumn{3}{|c|}{$\begin{array}{l}\text { Difference of coordinate } \\
\qquad(\mathrm{mm})\end{array}$} \\
\hline & $\Delta X$ & $\Delta Y$ & $\Delta Z$ & & $\Delta X$ & $\Delta Y$ & $\Delta Z$ \\
\hline 1 & 0.001 & -0.009 & 0.001 & 18 & -0.151 & -0.191 & 0.526 \\
\hline 5 & -0.192 & -0.053 & -0.158 & 21 & 0.041 & -0.180 & 0.530 \\
\hline 8 & 0.013 & -0.019 & 0.113 & 23 & -0.306 & -0.480 & 0.599 \\
\hline 10 & -0.174 & -0.097 & 0.138 & 25 & -0.738 & -0.582 & 0.685 \\
\hline 12 & -0.357 & -0.06 & -0.054 & 28 & -0.154 & -0.495 & 1.108 \\
\hline 13 & 0.001 & -0.001 & 0.387 & 31 & -0.796 & -0.746 & 1.051 \\
\hline
\end{tabular}

Table 3. Difference of check points object coordinates(three control points)

From Table 3, the mean square error of three directions are $m_{X}$ $= \pm 0.356 \mathrm{~mm}, m_{Y}= \pm 0.349 \mathrm{~mm}, m_{Z}= \pm 0.572 \mathrm{~mm}$ respectively, and the point mean square error is $m_{P}= \pm 0.759 \mathrm{~mm}$. Under this condition, we can not obtain the approximate values of exterior orientation elements by space resection.

\subsection{Experiment 2. using six control point}

Six images (shown in Figure 5.) are taken and their approximate values of exterior orientation elements are searched by PSO respectively. Twelve code points are selected as check points in the control brand, shown as in Figure 4(b). The exterior orientation elements calculated by PSO and Bundle adjustment are shown in Table 4 . The difference of check points object coordinates are shown in Table 5. 


\begin{tabular}{|c|c|c|c|c|c|c|c|}
\hline No & Method & $X_{S}(\mathrm{~mm})$ & $Y_{S}(\mathrm{~mm})$ & $Z_{S}(\mathrm{~mm})$ & $\begin{array}{c}\varphi \\
\text { (radian) }\end{array}$ & $\begin{array}{c}\omega \\
\text { (radian) }\end{array}$ & $\begin{array}{c}\kappa \\
\text { (radian) }\end{array}$ \\
\hline \multirow{2}{*}{1} & PSO & -86.16 & -68.67 & 2434.57 & 0.10740 & 0.08701 & -0.02690 \\
\cline { 2 - 8 } & Bundle & -437.22 & 248.62 & 3211.53 & 0.20354 & 0.03114 & -0.02434 \\
\hline \multirow{2}{*}{2} & PSO & -171.73 & -115.72 & 2446.62 & 0.13150 & 0.10620 & -0.04050 \\
\cline { 2 - 8 } & Bundle & -480.53 & -110.84 & 3144.28 & 0.21386 & 0.14294 & -0.04888 \\
\hline \multirow{2}{*}{3} & PSO & 393.60 & 18.27 & 2399.42 & -0.07580 & 0.05440 & 0.00240 \\
\cline { 2 - 8 } & Bundle & 402.07 & -110.92 & 3176.76 & -0.04708 & 0.14480 & -0.00399 \\
\hline \multirow{2}{*}{4} & PSO & 1020.67 & -61.73 & 2414.53 & -0.34640 & 0.07770 & 0.04720 \\
\cline { 2 - 8 } & Bundle & 1380.76 & -98.37 & 3082.11 & -0.37194 & 0.13500 & 0.05257 \\
\hline \multirow{2}{*}{5} & PSO & -184.12 & -224.49 & 2415.21 & -0.00880 & -0.03830 & -0.05280 \\
\cline { 2 - 8 } & Bundle & -544.01 & -463.28 & 3066.80 & 0.24614 & 0.25035 & -0.08283 \\
\hline \multirow{2}{*}{6} & PSO & 1227.60 & -140.33 & 2555.88 & -0.38700 & 0.10990 & 0.01540 \\
\cline { 2 - 8 } & Bundle & 1693.71 & -470.83 & 3118.71 & -0.44186 & 0.24396 & 0.03531 \\
\hline
\end{tabular}

Table 4. Exterior orientation elements calculated by PSO and Bundle adjustment (six control points)

\begin{tabular}{|c|c|c|c|c|c|c|c|}
\hline \multirow[t]{2}{*}{ No } & \multicolumn{3}{|c|}{$\begin{array}{c}\text { Difference of coordinate } \\
(\mathrm{mm})\end{array}$} & \multirow[t]{2}{*}{ No } & \multicolumn{3}{|c|}{$\begin{array}{l}\text { Difference of coordinate } \\
\qquad(\mathrm{mm})\end{array}$} \\
\hline & $\Delta X$ & $\Delta Y$ & $\Delta Z$ & & $\Delta X$ & $\Delta Y$ & $\Delta Z$ \\
\hline 1 & -0.013 & -0.013 & 0.009 & 18 & -0.128 & -0.032 & 0.336 \\
\hline 5 & -0.094 & -0.084 & -0.174 & 21 & 0.006 & 0.020 & 0.286 \\
\hline 8 & 0.0145 & -0.003 & 0.053 & 23 & -0.198 & -0.151 & 0.329 \\
\hline 10 & -0.072 & -0.066 & 0.065 & 25 & -0.386 & -0.160 & 0.393 \\
\hline 12 & -0.083 & -0.029 & -0.147 & 28 & -0.141 & -0.095 & 0.782 \\
\hline 13 & -0.011 & 0.040 & 0.271 & 31 & -0.413 & -0.145 & 0.685 \\
\hline
\end{tabular}

Table 5. Difference of check points object coordinates(six control points)

From Table5, the mean square error of three directions are $m_{X}=$ $\pm 0.187 \mathrm{~mm}, m_{Y}= \pm 0.089 \mathrm{~mm}, m_{Z}= \pm 0.373 \mathrm{~mm}$ respectively, and the point mean square error is $m_{P}= \pm 0.427 \mathrm{~mm}$. Under this condition, we can obtain the approximate values of exterior orientation elements by space resection, but the deviation to their real value are too large and lead to misconvergence of bundle adjustment.

\section{CONCLUSION}

By using PSO to calculate the approximate values of exterior orientation elements, the problem of unavailability of exterior orientation elements could be solved due to the decrease in the number of control points and narrow space distribution. The global searching ability of particle swarm optimization is very powerful. In terms of objective function based on the few controlling points and the set gross area of exterior orientation elements, the approximate values of exterior orientation elements can be solved and used in bundle adjustment. This method can be applied in real production and experiments, which can reduce the procedure of surveying control points and increase surveying efficiency.

\section{REFERENCE}

Feng, W.H., 2003. Close-range Photogrammetry. Wuhan University Press, Wuhan.

Guan, Y.L., Cheng, X.J., Zhou, S.J., Liao, Z.P., Lu, T.D., 2008. A Solution to space resection based on unit quaternion. Acta Geodaetica et Cartographica Sinica, 37(1), pp. 30-35.

Haralick, R.M., Lee, C.-N., Ottenberg, K. \& Nlle, M., 1994. Review and analysis of solutions of the three point perspective pose estimation problem. International Journal of Computer Vision,13 (3), pp.331-356

Kennedy, J., Eberhart, P., 1995. Particle swarm optimization. In: Proceedings of IEEE International Conference on Neural Networks, Perth, Australia,Vol4, pp. 1942-1948.

Parsopoulos, K.E.,Plagianakos, V.P., Magoulas, G.D., Vrahatis, M.N., 2001. Advances in Convex Analysis and Global Optimization. Kluwer Academic Publishers, Dordrecht, The Netherlands, pp. 445-457.

Reynolds, C.W., 1987. Flocks, herds and schools: a distributed behavioral model. Computer Graphics (Proceedings of SIGGRAPH 87), 21 (4), pp. 25-34.

Shi, Y., Eberhart, R.C., 1998. A modified particle swarm optimizer. In: Proceedings of IEEE International Conference of Evolutionary Computation, Anchorage, Alaska, pp. 69-73.

Shi, Y., Eberhart, R.C., 1999. Empirical study of particle swarm optimization. In: Proceedings of IEEE Conference of Evolutionary Computation, Washington DC, USA, pp. 19451950.

Wang, B.F., Gu,H.C., Huang, G.P., Chen, J.H., 2006. Method of calculating synoptical elements of exterior orientation. Journal of Geomatics Science and Technology, 23(3), pp. 182-184.

Wei, B., Lin, C., Zheng, Y.K., 2008. Experiment and analysis of parameters in particle swarm optimization. Journal of Xihua University(Natural Science Edition), 27(1), pp. 76-80.

Zeng, J.C., Jie, Q., Cui, Z.H., 2004. Particle Swarm Optimization. Science Press, Beijing, pp. 9-13.

Zhang, Y.J, Zhang, Z.X., Zhang, J.Q., 2002.Camera calibration using 2D-DLT and bundle adjustment with planar scenes. Geomatics and Information Science of Wuhan University, 27(6), pp. 566-571. 